\title{
Correction to: Small strain multiphase-field model accounting for configurational forces and mechanical jump conditions
}

Daniel Schneider ${ }^{1,2} \cdot$ Ephraim Schoof ${ }^{2}$. Oleg Tschukin ${ }^{1} \cdot$ Andreas Reiter $^{1} \cdot$ Christoph Herrmann $^{2} \cdot$ Felix Schwab $^{1}$. Michael Selzer ${ }^{1,2} \cdot$ Britta Nestler ${ }^{1,2}$

Published online: 7 December 2017

(c) Springer-Verlag GmbH Germany, part of Springer Nature 2017

\section{Correction to: Comput Mech}

\author{
https://doi.org/10.1007/s00466-017-1458-4
}

In the original publication, Eq. 52 was published incorrectly.

The correct equation should read as follows:

$$
\begin{aligned}
\llbracket p\left(\boldsymbol{\sigma}_{n}, \boldsymbol{\varepsilon}_{t}\right) \rrbracket & =\llbracket W \rrbracket-\boldsymbol{\sigma}_{n} \cdot \llbracket \boldsymbol{\varepsilon}_{n} \rrbracket \\
& =\llbracket W \rrbracket-\boldsymbol{\sigma} \boldsymbol{n} \cdot \llbracket \boldsymbol{\varepsilon} \rrbracket \boldsymbol{n}=-\gamma_{\alpha \beta} \kappa .
\end{aligned}
$$

The original article was corrected.

The original article can be found online at https://doi.org/10.1007/ s00466-017-1458-4.

Daniel Schneider

daniel.schneider@kit.edu

Ephraim Schoof

ephraim.schoof@hs-karlsruhe.de

Oleg Tschukin

oleg.tschukin2@kit.edu

Andreas Reiter

andreas.reiter@kit.edu

Christoph Herrmann

christoph.herrmann2@hs-karlsruhe.de

Felix Schwab

felix.schwab@kit.edu

Michael Selzer

michael.selzer@kit.edu

Britta Nestler

britta.nestler@kit.edu

1 Institute of Applied Materials (IAM-CMS), Karlsruhe

Institute of Technology (KIT), Straße am Forum 7, 76131

Karlsruhe, Germany

2 Institute of Materials and Processes (IMP), Karlsruhe

University of Applied Sciences, Moltkestrasse 30, 76133

Karlsruhe, Germany 